\title{
Dynamic response functions for the Holstein-Hubbard model
}

\author{
W. Koller, ${ }^{*}$ D. Meyer, ${ }^{\dagger}$ and A. C. Hewson ${ }^{\ddagger}$ \\ Department of Mathematics, Imperial College, London SW7 2BZ, UK
}

(Dated: 30 March 2004)

\begin{abstract}
We present results on the dynamical correlation functions of the particle-hole symmetric HolsteinHubbard model at zero temperature, calculated using the dynamical mean field theory which is solved by the numerical renormalization group method. We clarify the competing influences of the electron-electron and electron-phonon interactions particularity at the different metal to insulator transitions. The Coulomb repulsion is found to dominate the behaviour in large parts of the metallic regime. By suppressing charge fluctuations, it effectively decouples electrons from phonons. The phonon propagator shows a characteristic softening near the metal to bipolaronic transition but there is very little softening on the approach to the Mott transition.
\end{abstract}

PACS numbers: 71.10.Fd,71.30.+h,71.38.-k

\section{INTRODUCTION}

Phonons are important in metallic systems. They affect the electronic behaviour in diverse ways causing the dominant temperature dependent contribution to the resistivity, an enhancement of the specific heat, and an attractive effective electron-electron interaction, which may induce superconductivity. One can expect strong electron-phonon interactions in strongly correlated systems, such as heavy fermion compounds ${ }^{1}$, where the radius of the rare earth ions is very sensitive to the $f$ electron occupation. The coupling of electronic states to the lattice also plays an important role in the anomalous electronic behaviour of the manganites ${ }^{2-4}$. The superconductivity in the fullerides ${ }^{5}$ appears to be induced by electron-phonon interactions within a strongly correlated electron band.

However, the fully quantum mechanical treatment of lattice effects in these compounds has so far received comparatively little attention. The reason for that lies in the difficulty in handling strong electron-phonon coupling together with strong electron-electron repulsion. Recent advances in non-perturbative techniques, most notably the development of the dynamical mean field theory $(\mathrm{DMFT})^{6}$, enable one to investigate the interplay of electron and phonon interactions in these systems.

One of the most prominent models in the field of strongly correlated electron systems is the Hubbard model $^{7}$ which been used extensively to study the effects of strong local electron-electron interactions. Especially within the framework of $\mathrm{DMFT}^{6}$ the nature of the Mott transition could be clarified ${ }^{8,9}$. The Holstein model ${ }^{10}$ has been used to study polaronic effects in the absence of electron-electron repulsion, mainly in the limit of low electron density. Systems with finite electron density have received less attention, but have been studied within the DMFT using Monte Carlo ${ }^{11}$, exact diagonalization $(\mathrm{ED})^{12}$, perturbative approaches ${ }^{13-15}$ and the numerical renormalization group $(\mathrm{NRG})^{16}$.

Even less studied has been the interplay of electron-electron interactions and the electron-phonon coupling ${ }^{17-21}$. The most natural model incorporating

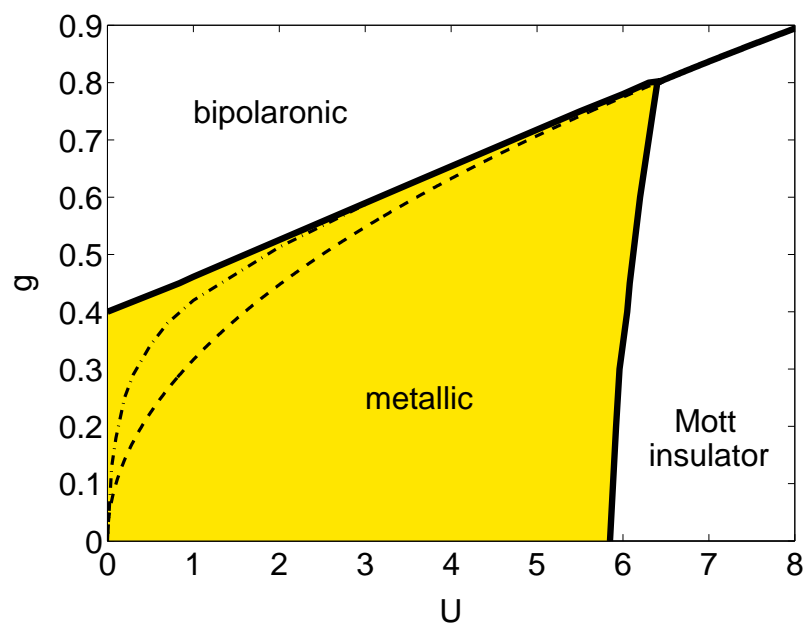

FIG. 1: Zero temperature phase diagram of the half filled Holstein-Hubbard model for $\omega_{0}=0.2$ and a semielliptic band of width $W=4$ as calculated in Ref. 22 . The dashed line is the locus of $U_{\text {eff }}=0$. Points on the dot-dashed line fulfil $\left\langle n_{\uparrow} n_{\downarrow}\right\rangle=1 / 4$.

both these terms is the Holstein-Hubbard model defined by the Hamiltonian

$$
\begin{aligned}
H= & \sum_{\vec{k} \sigma} \epsilon(\vec{k}) c_{\vec{k} \sigma}^{\dagger} c_{\vec{k} \sigma}+U \sum_{i} n_{i \uparrow} n_{i \downarrow} \\
& +\omega_{0} \sum_{i} b_{i}^{\dagger} b_{i}+g \sum_{i}\left(b_{i}^{\dagger}+b_{i}\right)\left(n_{i \uparrow}+n_{i \downarrow}-1\right) .
\end{aligned}
$$

Here $U$ describes the electron-electron interaction within a band of dispersion $\epsilon(\vec{k})$. The electron density $n_{i \uparrow}+$ $n_{i \downarrow}$ at site $i$ couples linearly to the local displacement operator $x_{i} \equiv\left(b_{i}+b_{i}^{\dagger}\right) / \sqrt{2 m \omega_{0}}$ with an electron-phonon coupling $g$. The phonons are assumed to be dispersionless (local Einstein phonons) with energy $\omega_{0}$ and $m$ is the mass of the vibrating ions.

Recently, the $T=0$ phase diagram of the half filled Holstein-Hubbard model has been calculated ${ }^{22,23}$ using various local approximations, and is shown in Fig. 1. The results are for a semi-elliptic band of width $W=4$ 
and phonon frequency $\omega_{0}=0.2(m=1)$. All types of long-range order are excluded. We can distinguish three different phases: metallic, bipolaronic and Mott insulating phase. The metallic phase is always found to be a Fermi liquid. The latter two phases display a gap in the one-electron spectra and will be referred to as gapped phases. The parameters of Fig. 1, namely $W=4, m=1$ and $\omega=0.2$ are taken throughout this paper, unless mentioned otherwise.

One way of simplifying the discussion of the HolsteinHubbard model would be to integrate out the phonons in order to derive an effective model for the electrons. The effective action is then governed by the $\omega$-dependent potential $^{24}$

$$
U_{\mathrm{eff}}(\omega)=U+\frac{2 g^{2} \omega_{0}}{\omega^{2}-\omega_{0}^{2}}
$$

This reduces to the static quantity $U_{\text {eff }} \equiv U-2 g^{2} / \omega_{0}$ in the limits $\omega \rightarrow 0$ and $\omega_{0} \rightarrow \infty$. Therefore, on small energy scales, we expect an effective attractive or repulsive interaction depending on the value of $U_{\text {eff. }}$ For small phonon energies $\omega_{0}$, however, there is no reason to expect the static quantity $U_{\text {eff }}$ to be sufficient to characterize the behaviour of the Holstein-Hubbard model. The properties of this model are expected to depend on both $U$ and $g$ individually. This is also apparent from the phase diagram in Fig. 1. The dot-dashed line, where the double occupancy takes the value of a free system $\left\langle n_{\uparrow} n_{\downarrow}\right\rangle=1 / 4$ is quite distinct from the line $U_{\mathrm{eff}}=0$. The aim of this study is to clarify the interplay of these different types of interactions.

An interesting question about this model is whether the suppression of local charge fluctuations by a significant Hubbard $U$ will effectively decouple electrons and phonons on all energy scales. It is possible, however, that for the behaviour on low energy scales the effective onsite- $U$ is strongly renormalized and hence the electron phonon coupling will have a comparatively stronger effect on energy scales $\omega<\omega_{0}$ (see Ref 17,20).

In this paper, we present results on dynamical electronic and phonon response functions for the HolsteinHubbard model as calculated within the dynamical mean field theory ${ }^{6}$ (DMFT). The effective impurity problem is solved using Wilson's numerical renormalization group (NRG) method ${ }^{25}$ extended to treat the electron-phonon coupling $^{24}$. The combination of DMFT and NRG has proven to be a reliable and effective method for calculations of both metallic and insulating phases at $T=0$. It has been applied to the Mott transition in the pure Hubbard model ${ }^{26}$ and to the pure Holstein model ${ }^{16}$. In our calculations we use the discretization parameter $\Lambda=1.8$ and retain up to 1200 states. The value of $\Lambda=1.8$ gives rise to a bandwidth correction factor $A_{\Lambda}=1.029$ (see Eq. (5.42) in 25) which we take into account in the NRG procedure. For the calculation of dynamical response functions we use the method described in Ref. 27.

Apart from the usual local one-electron Green's function $G_{\sigma}(\omega) \equiv\left\langle\left\langle c_{i \sigma} ; c_{i \sigma}^{\dagger}\right\rangle\right\rangle_{\omega}$ we will be interested in two different phonon Green's functions,

$$
\begin{aligned}
d(\omega) & =\left\langle\left\langle b_{i} ; b_{i}^{\dagger}\right\rangle\right\rangle_{\omega}, \\
D(\omega) & =2 \omega_{0}\left\langle\left\langle x_{i} ; x_{i}\right\rangle\right\rangle_{\omega}=\left\langle\left\langle b_{i}+b_{i}^{\dagger} ; b_{i}+b_{i}^{\dagger}\right\rangle\right\rangle_{\omega} .
\end{aligned}
$$

Since we will treat only homogeneous phases here and exclude all types of long-range order, we have dropped the site indices in all of the definitions. The spectral weight of $d(\omega)$ will be denoted by $\rho_{d}(\omega) \equiv-\frac{1}{\pi} \operatorname{Im} d(\omega+$ $\left.i 0^{+}\right)$, and similar for all other Green's functions. The dynamical charge and spin susceptibility are given by

$$
\begin{aligned}
& \chi_{c}(\omega)=\langle\langle\hat{O} ; \hat{O}\rangle\rangle_{\omega}, \\
& \chi_{s}(\omega)=\left\langle\left\langle n_{i \uparrow}-n_{i \downarrow} ; n_{i \uparrow}-n_{i \downarrow}\right\rangle\right\rangle_{\omega},
\end{aligned}
$$

respectively. The operator $\hat{O}$ represents the electronic term that couples to the phonons,

$$
\hat{O}=n_{i \uparrow}+n_{i \downarrow}-1 .
$$

It should be noted that our definition of $\chi_{s}(\omega)$ differs from the usual one by a factor of four. We also calculate the quasiparticle weight $z$ given by $z=\left(1-\operatorname{Re} \Sigma^{\prime}(0)\right)^{-1}$ within the local approximation of the DMFT.

The different regimes of the phase diagram of Fig. 1 give rise to the following structure of the paper: In Sec. II we choose a fixed value of $U$ and study dynamical properties as a function of the phonon coupling $g$. We find continuous transitions from a metallic to a bipolaronic state for small values of $U=0$ and $U=1$ (Sec. II A). For a larger value of $U=5$, as discussed in Sec. II B, the transition to the bipolaronic state becomes discontinuous. Complementarily, we look at the $U$-dependence of dynamical properties in Sec. III keeping $g$ fixed to $g=0.45$. For this value of $g$ the system can be in all three phases, depending on $U$. In Sec IV we study the properties of the model on the two polaronic lines as given by $U_{\text {eff }}=0$ and $\left\langle n_{\uparrow} n_{\downarrow}\right\rangle=1 / 4$ (dashed and dot-dashed lines in Fig. 1). We also show spectra for $U_{\text {eff }}=0$ for different values of $\omega_{0}$. In Sec. $V$ we give an overview of our results, and we present our conclusions in Sec. VI.

\section{DEPENDENCE ON $g-$ PHONON DRIVEN PHASE TRANSITION}

In this section, we fix the value of the Hubbard interaction $U$ and study dynamical properties for a varying electron-phonon coupling $g$. We distinguish between the cases of weak and strong $U$.

\section{A. Weak $U$}

First of all, we look at the one-electron spectra for a fixed $U=1$ as shown in Fig. 2. There is a strong similarity of these results with those of the pure Holstein model $(U=0)$ calculated previously ${ }^{16}$. At weak coupling 


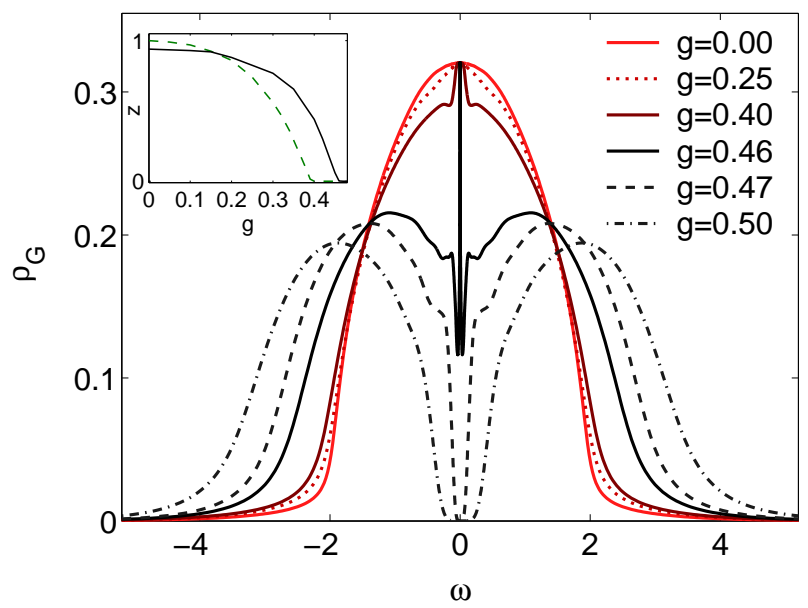

FIG. 2: One-particle spectral function for $U=1$ and various values of $g$. The inset shows the variation of the quasiparticle weight $z$ for $U=1$ (solid line) and $U=0$ (dashed line).

a narrow peak emerges at the Fermi energy. The top of this feature is rather flat and the imaginary part of the self energy has a shallow $\omega^{2}$-dependence. This one would expect from lowest order perturbation theory in $g$, where the imaginary part of the self energy vanishes in the range $|\omega|<\omega_{0}$ for $U=0$ (see Eq. (19) in Ref. 24). Upon increasing $g$ further, there is a rapid narrowing of this feature until it disappears at a critical coupling of $g_{c} \approx 0.47$ and a gap opens. Similar to the pure Holstein model, there is no preformed gap in this case in contrast to the Mott transition in the Hubbard model.

The metallic regime corresponds to a renormalized Fermi liquid. The quasiparticle weight $z$ is shown as a function of $g$ in the inset together with that for the pure Holstein model $(U=0)$. For $U=1$, the quasiparticles are already slightly renormalized at $g=0$. However, initially the renormalization with $g$ is weaker than in the case $U=0$ but more rapid on the approach to $g_{c}{ }^{22}$. As with the pure Holstein model, there is no evidence of multiple solutions near the phase transition. This is in contrast to the situation near the Mott transition for the Hubbard model, where there is a finite coexistence region.

Figure 3 shows the spectra of the corresponding charge- and spin susceptibilities at $U=1$ and $U=0$ for comparison. In the free system, $\chi_{c}(\omega)=\chi_{s}(\omega)$ as defined in Eq. (4), which can be seen in the lowest curves of the two upper panels. Looking at the case $U=0$ first (upper panel), we see the emergence and buildup of a low-energy peak in $\chi_{c}$ and the suppression of weight in $\chi_{s}$ for $\omega<W / 2$. In the bipolaronic state $(g=0.42)$, both charge and spin susceptibilities have the same gap, roughly twice that seen in the electronic spectrum. They have an identical peak above this gap. These can be seen in the dotted curves of Fig. 3, which have been scaled up by a factor of 10 to make these features visible. The coincidence of these peaks can be explained by the fact
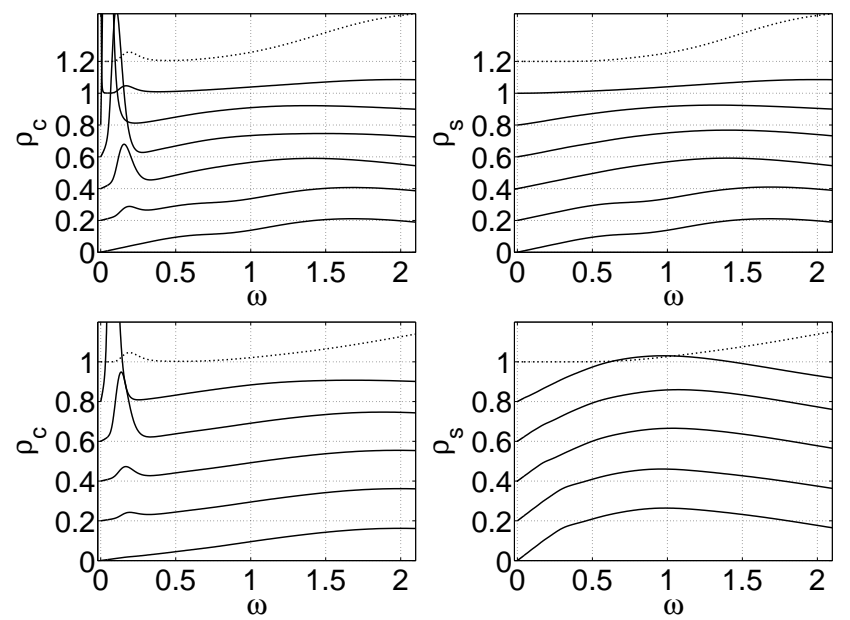

FIG. 3: Low energy behaviour of the spectra of the charge (left) and spin-susceptibility (right) for the pure Holstein model ( $U=0$, top) and $U=1$ (bottom). The values of $g$ are $g=0.0$ (bottom), $g=0.1,0.2,0.3,0.37,0.40,0.42$ (top) in the upper panel and $g=0.0$ to 0.5 in steps of 0.1 in the lower panel. The dotted lines are scaled up by a factor of 10 .

that spin fluctuations necessarily require the breakup of a bipolaronic pair. Their weight is very small due to the strong bipolaronic binding. There is an additional very small peak in the charge susceptibility in the gap close to $\omega_{0}$ from the residual couplings to the phonon mode. In the absence of charge ordering, there must be charge fluctuations at $\omega=0$ which, however, cannot be observed numerically.

For $U=1$ (lower panel) we observe the same overall trends. However, starting from a reduced charge susceptibility due to the finite $U$, the peak in $\chi_{c}$ develops more slowly. The spin susceptibility, which is enhanced due to the finite $U$, changes very little in the metallic phase. The low-energy spin fluctuations are almost completely suppressed with the emergence of the gap in the bipolaronic state.

The charge fluctuations can be directly related to the phonon propagator via the equation (see appendix A)

$$
d(\omega)=d_{0}(\omega)+g^{2} d_{0}(\omega) \chi_{c}(\omega) d_{0}(\omega)
$$

where $d_{0}(\omega)=1 /\left(\omega-\omega_{0}\right)$ is the free phonon propagator. However, use of this equation with an approximate form of $\chi_{c}(\omega)$ can lead to severe numerical errors, as discussed in appendix A. In Fig. 4, we give the results for the phonon spectra for $U=0$ and $U=1$ calculated via the self energy ${ }^{28}$, similar to the procedure we use for the electronic spectra ${ }^{27}$. The results for $U=0$ differ significantly from those published earlier ${ }^{16}$ based on Eq. 6 .

In contrast to the earlier results, the main phonon peak is found initially to soften significantly in a similar way to the Migdal Elisahberg result. The two-peak structure develops only upon approaching the transition to the bipolaronic state. In the gapped state, the high-frequency peak narrows and tends towards $\omega_{0}$. The same trend 

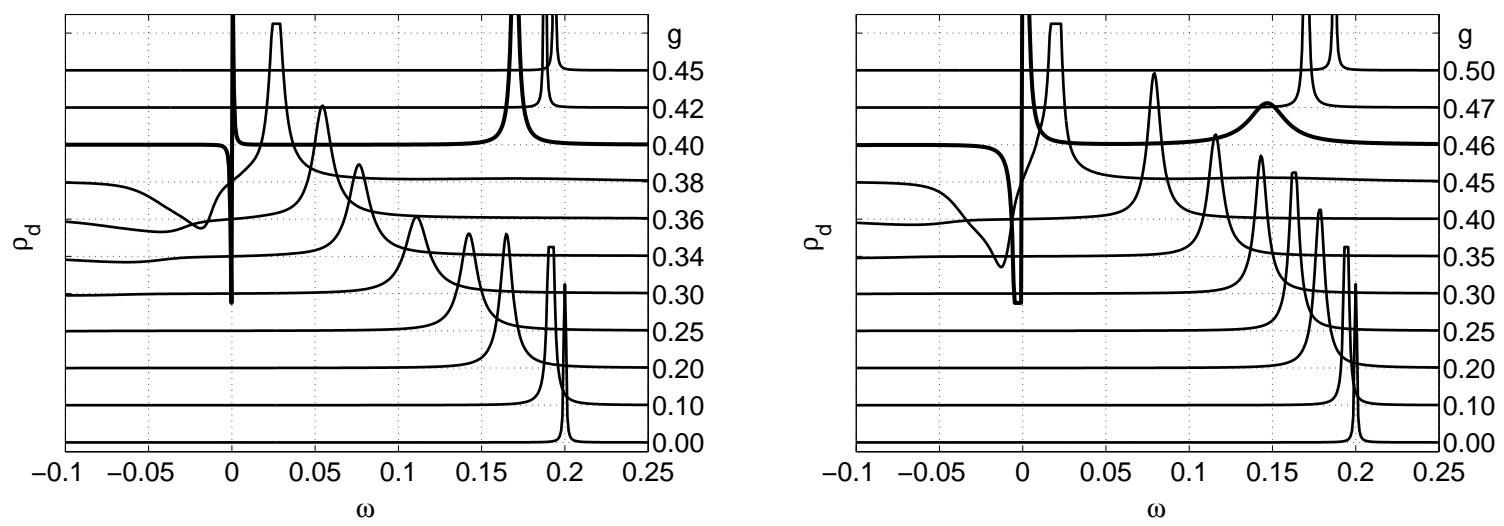

FIG. 4: Spectral density of the phonon propagator for $U=0$ (left) and $U=1$ (right) and various values of $g$.

can be seen for $U=1$ (right-hand plot). The slower initital softening correlates with the suppression of charge fluctuations seen in $\chi_{c}(\omega)$.

On the approach to the transition, significant negative spectral weight for $\omega<0$ can be seen in the spectra shown in Fig. 4. As a consequence of the spectral theorem, this relates directly to the average number of excited phonons in the ground state. The spectra of the Green's function $D(\omega)$ show similar features as $d(\omega)$, and integrated up to $\omega=0$ yield the average lattice fluctuations as shown in Fig. 5. These results agree well with the direct evaluation of the expectation value $\left\langle\left(b+b^{\dagger}\right)^{2}\right\rangle$ in the metallic phase. For low to intermediate values of $g$, the NRG results do not deviate significantly from the Midgal Eliashberg calculation and are significantly smaller than reported in Ref. 16 based on Eq. 6. On the ap-

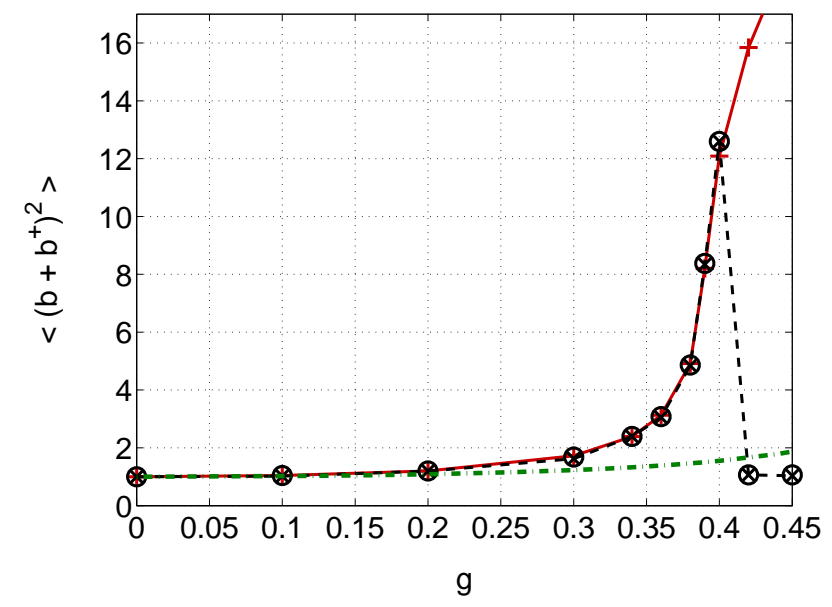

FIG. 5: Expectation value of the lattice fluctuations as a function of the electron-phonon coupling $g$ in the pure Holstein model $(U=0)$. In the metallic range $(g<0.4)$, the direct calculation $(+)$ agrees well with the integration over the phonon Green's function (o) and charge susceptibility $(\times)$ ). For details see appendices A and B. The dot-dashed line shows the result from the Migdal Eliashberg calculation. proach to the transition, there is a large increase in the lattice fluctuation. In the gapped phase, we observe a large difference between the direct calculation and the integration over the negative part of the phonon spectral density. One way of looking at it is that due to the limited numerical resolution we miss the contribution from the peak at $\omega=0^{-}$in the spectrum of $D(\omega)$. An other way of explaining this difference is that there is no dynamics connecting the two degenerate ground states with $\langle n\rangle=0$ and 2 in the gapped phase. These two ground states have nonvanishing average displacements $\pm x_{0}$, see appendix B, and integration over the phonon spectrum yields the fluctuations about $\pm x_{0}$, i.e., $\left\langle\left(x-x_{0}\right)^{2}\right\rangle$. The calculation of $x_{0}$ in one of the ground states quantitatively explains the different values of the two methods in the gapped phase.

\section{B. Strong $U$}

Next we look at the transition to the bipolaronic state for a larger fixed value of $U=5$. Figure 6 shows the corresponding one-particle electron spectral functions for various values of $g$. Their $g$-dependence is in sharp contrast to the small $U$ case shown in Fig. 2. Initially for $g=0$, we have the three-peak structure of a strongly correlated Hubbard model. With increasing $g$, the central resonance broadens slightly as is reflected in the increasing quasiparticle weight $z$ (see inset). This can be ascribed to the partial cancellation of the Hubbard repulsion by phonon mediated electron-electron attraction, see Eq. (2). The effect, however, is much weaker than one would deduce from $U_{\text {eff }}$. The position of the high-energy Hubbard bands is virtually unaffected by the phonons, as expected from Eq. (2) evaluated at $\omega \approx \pm U / 2$. When approaching the critical coupling $g_{c} \approx 0.72$, the quasiparticle peak disappears abruptly and $z$ jumps to zero, indicating a discontinuous transition to the bipolaronic phase. The double occupancy, shown in the inset to Fig. 6, is much less than a quarter in the metallic state 


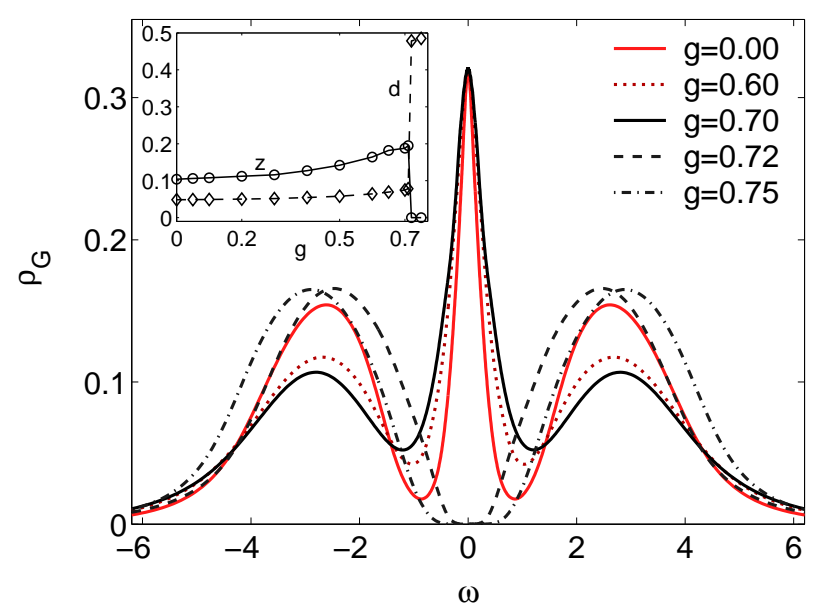

FIG. 6: One-particle spectral function for $U=5$ and various values of $g$. The central peak broadens with increasing $g$ and at $g \approx 0.71$ disappears in a first order phase transition to the bipolaronic state. The inset shows the quasiparticle weight $z$ (o) and the double occupancy $d=\left\langle n_{\uparrow} n_{\downarrow}\right\rangle(\diamond)$ as functions of $g$.

but makes a sudden jump to $d \approx 1 / 2$ on the point of the phase transition. A coexistence of metallic and gapped solutions is found in the narrow range $0.71 \lesssim g \lesssim 0.72$.

The spectra of the charge and spin susceptibilities are shown in Fig. 7. They display similar trends, but more pronounced, than the corresponding results for $U=1$ (lower panels of Fig. 3). The low-energy peak in $\chi_{c}(\omega)$ only becomes clearly visible when $g$ reaches the value of $g \approx 0.65$. In the bipolaronic phase, the peaks above the gap in the charge and spin susceptibilities are not identical as in the $U=0$ case, but very similar. However, the gaps are too large to be visible on the $\omega$-scale of Fig. 7. The only peak visible, when enhanced by a factor of 20 , is the peak in the charge susceptibility within the gap near $\omega_{0}$.

In Fig. 8, the spectra of the corresponding phonon propagators are plotted. Over a large initial range of $g \lesssim 0.6$ there is very little softening, corresponding to a complete suppression of charge fluctuations. When approaching $g_{c}$, the phonon mode softens and develops a
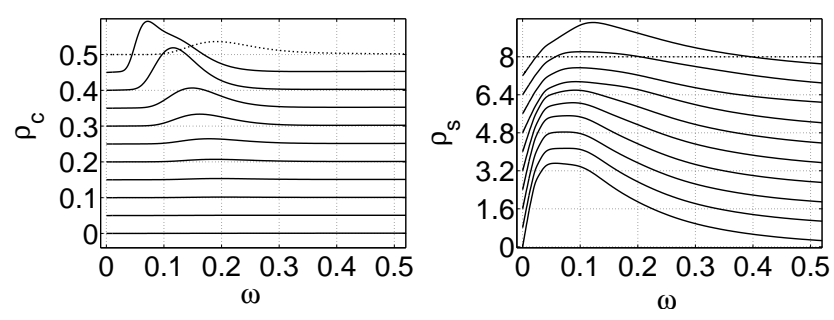

FIG. 7: Low-energy behaviour of the spectra of the charge (left) and spin-susceptibility (right) for $U=5$. The values of $g$ correspond to those in Fig. 8. Note the different scales of the $y$-axes. Dotted lines have been scaled up by a factor of 10 .

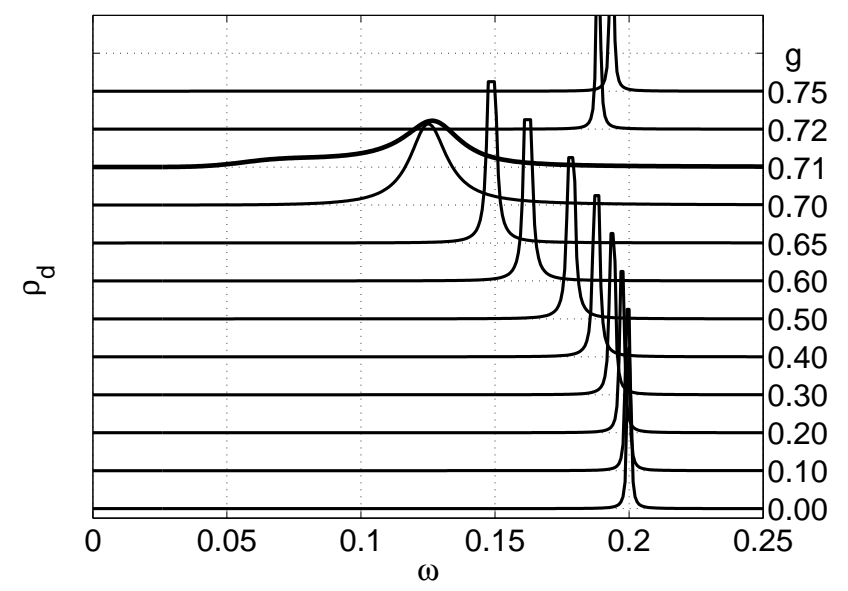

FIG. 8: Spectral density of the phonon propagator for $U=5$ and various values of $g$.

low-energy shoulder. After the discontinuous transition to the bipolaronic phase, the mode hardens back to $\omega_{0}$ and narrows.

\section{DEPENDENCE ON $U$ - MOTT TRANSITION}

In this section we will fix the electron-phonon coupling at the value $g=0.45$ and study the dependence of dynamic response functions on the Hubbard repulsion $U$. The value of $g=0.45$ is chosen because all three phases can be found for it, depending on $U$ (see Fig. 1).

Figure 9 shows the one-electron spectral function in the vicinity of both phase transitions. The right-hand plot for large values of $U$ is very similar to what is observed in the Mott transition of the pure Hubbard model. The lower and the upper Hubbard peak at $\pm U / 2$ are well developed and move to higher energies as $U$ increases. At the same time, the central peak narrows and vanishes at the Mott transition. As for the pure Hubbard model, there is a preformed gap in the one-electron spectrum and a broad region of numerical coexistance of metallic and insulating solutions. The electronic spectra are very similar to those of the pure Hubbard model near the Mott transition. The phonons do not seem to alter much the picture of the phase transition.

The transition from the bipolaronic to the metallic state is completely different, as can be seen in the lefthand plot of Fig. 9. The phase transition occurs close to $U=1$ and the spectra show a similar behaviour as discussed in Sec. II A, where $g$ is varied. In the bipolaronic state, with the initial increase of $U$, the two polaron bands move towards the Fermi level. On the metallic side, just after the transition, we see a very sharp resonance which broadens when $U$ is increased further. There is no signature of a preformed gap once the system is metallic. Again, there is no evidence of a significant co- 

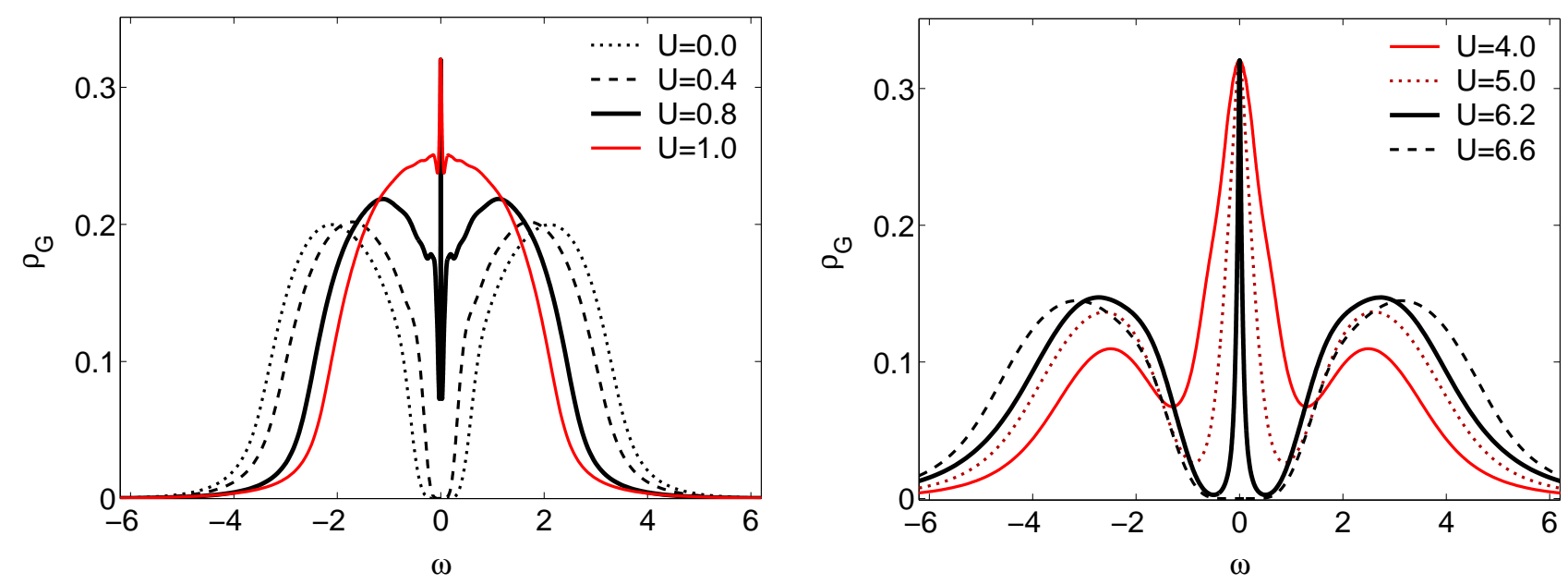

FIG. 9: One-particle spectral function for $g=0.45$ and values of $U$ close to the transition from the bipolaronic to the metallic state (left) and $U$ close to the transition to the Mott insulator (right).

existence region.

The spectra of the charge and spin susceptibilities for $g=0.45$ are shown in Fig. 10. We clearly see that the low-energy features of the two susceptibilities signal the appearance of two different instabilities of the system, depending on $U$. In the bipolaronic phase, both spectra are gapped and we see only the peak near $\omega_{0}$ in the charge susceptibility. Upon entering the metallic phase, the zero frequency peak in the charge susceptibility moves to finite $\omega$. This peak loses weight and slightly disperses to higher energies as the system becomes more metallic. In this small $U$ regime, spin fluctuations are suppressed.

The converse occurs when approaching the Mott transition at $U \approx 6.2$. The charge fluctuations are suppressed and peak in the spin susceptibility builds up, signalling instability towards antiferromagnetic ordering. In the Mott insulating state, both susceptibilities are gapped. The spin fluctuations above the gap (beyond the scale of Fig. 10) must largely be due to the charge fluctuations, as in the pure Hubbard case. However these peaks are not identical, in contrast to the bipolaronic gapped state. The difference in the peaks can be interpreted as a result of the spin correlations induced by $U$. However, due to the phonon coupling the spin fluctuation peak is reduced compared to the pure Hubbard case.

Figure 11 displays the phonon propagator for this scan. In the bipolaronic phase at $U=0$, electrons and phonons are almost decoupled, and the phonon spectrum shows only one peak just below $\omega_{0}=0.2$. When increasing $U$, this mode softens and splits into two peaks close to the phase transition. On the metallic side, the low-energy peak survives and continuously hardens back to $\omega_{0}$, as the system approaches the Mott transition. There is no signature of the Mott transition in the phonon spectrum. The effect of an increasing $U$ is just to suppress continuously the charge fluctuations which results in a decoupling of electrons and phonons which drives the hardening of the phonon peak.
Another feature of the suppression of charge fluctuations is the narrowing of the phonon peak with increase of $U$, as also observed in Ref. 18. In our case, however, the shift of the peak appears to be more marked. The narrowing can also be seen in comparing the curves with the same (low) value of $g$ in Figs. 4 and 8.

Further insight into the nature of these transitions can be gained by looking at the behaviour of the higher order electron Green's function

$$
F_{\sigma}(\omega)=\left\langle\left\langle c_{\sigma} c_{\bar{\sigma}}^{\dagger} c_{\bar{\sigma}} ; . c_{\sigma}^{\dagger}\right\rangle\right\rangle_{\omega}
$$

This Green's function is needed for the calculation of the self energy in the NRG procedure ${ }^{27}$. The integrated spectral weight for $-\infty<\omega<0$ equals the double occupancy $\left\langle n_{\uparrow} n_{\downarrow}\right\rangle^{27}$. Moreover, the total spectral weight of $F_{\sigma}(\omega)$ yields the average density $\left\langle n_{\bar{\sigma}}\right\rangle$, which should be $\left\langle n_{\uparrow}\right\rangle=\left\langle n_{\downarrow}\right\rangle=0.5$ in the particle-hole symmetric case treated here.

Figure 12 shows the spectral function of $F(\omega)$ for $g=0.45$. In the bipolaronic phase, almost all of the weight is located below the Fermi energy signalling a high value of the double occupancy (see inset). As we enter the metallic phase, a large part of this weight is transferred to higher energies. In the Mott insulator, virtually no weight is left at $\omega<0$ and the double occupancy is completely suppressed.

The inset to Fig. 12 also shows the total spectral weight of $F(\omega)$, which should evaluate to $1 / 2$. We see that this is indeed more or less the case, except for values of $U$ close to the phase transitions $U \approx 1$ and $U \approx 6$. For these values, the truncation of the Hilbert space in the NRG introduces the most significant errors. There we also find the strongest discrepancies between the direct calculation of the double occupancy from the ground state expectation value (marked with $x$ ) and the indirect, less accurate calculation via $F(\omega)$ (marked with $\diamond)$. 

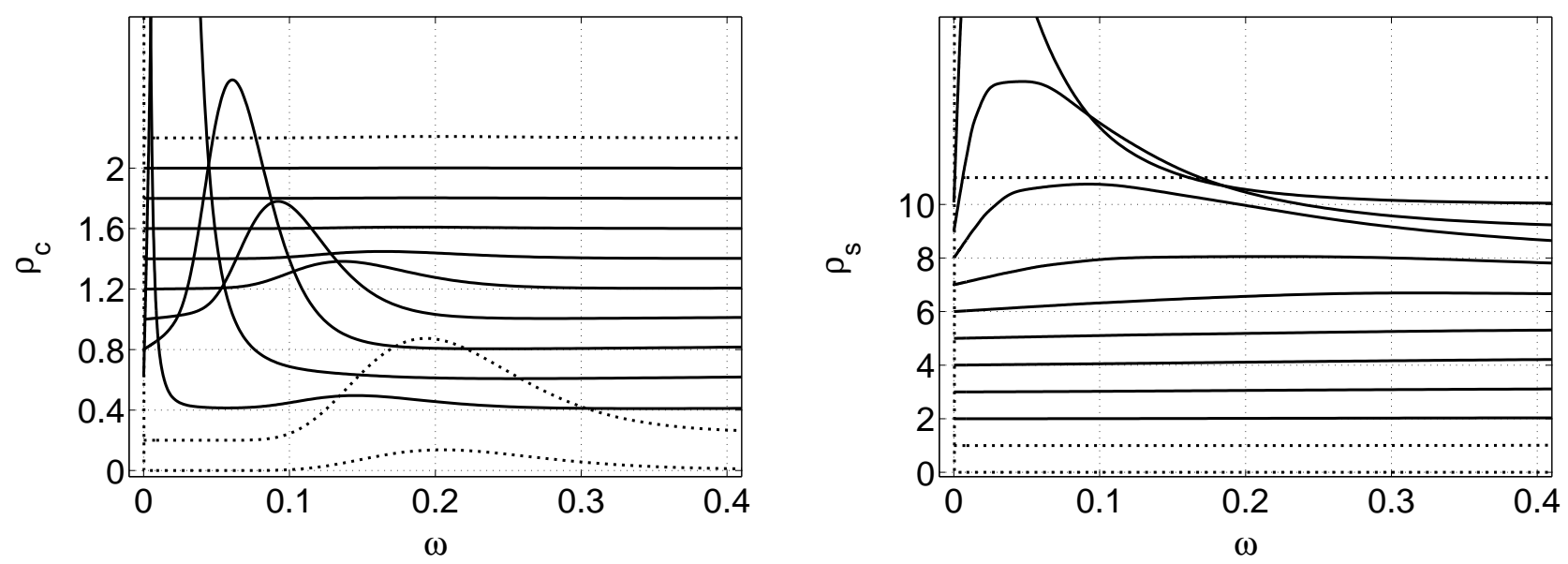

FIG. 10: Spectra of the charge (left) and spin-susceptibility (right) for $g=0.45$ and various values of $U$ (same as in Fig. 11). The charge susceptibility reflects the transition from a bipolaronic state to a metal, whereas the spin susceptibility signals the transition to the Mott insulator. The dotted lines are scaled up by a factor of 100 to show the peak at $\omega=\omega_{0}$ in the bipolaronic phase.

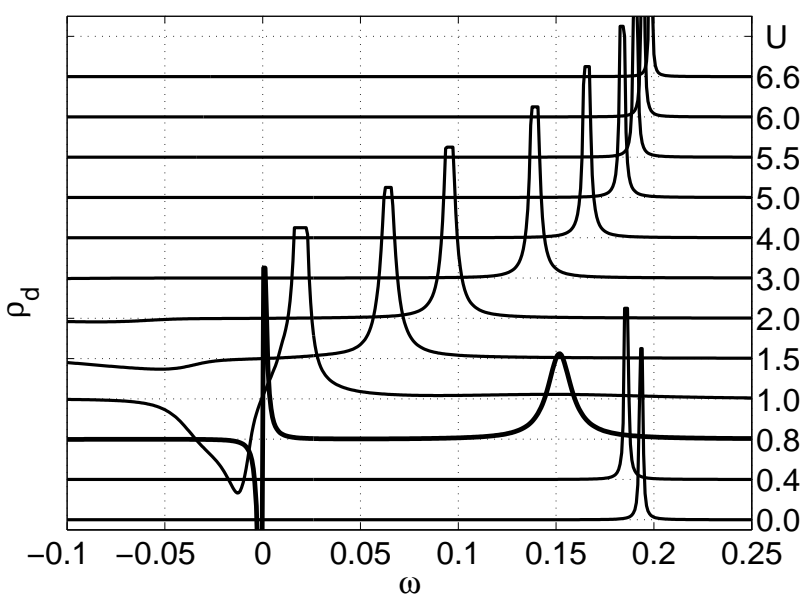

FIG. 11: Spectral density of the phonon propagator for $g=0.45$ and various values of $U$. The transition from the bipolaronic to the metallic state is clearly visible by the twopeak structure whereas the transition to the Mott insulator does not obviously affect the phonon propagator.

\section{POLARONIC LINES}

In this section we discuss the dynamical properties along two particular lines in the $g-U$ plane (see Fig. 1). The first of these lines is given by $U_{\text {eff }}=U+2 g^{2} / \omega_{0}=0$ where one might naively expect free quasiparticles close to the Fermi surface. The other line is the location of points where the double occupancy $\left\langle n_{\uparrow} n_{\downarrow}\right\rangle=1 / 4$, as in the free system.

Only for very large values of $\omega_{0}$ can the Hubbard term be completely cancelled by the phonon-mediated attractive term (see Eq. 2), in which case both polaronic lines would coincide. We investigate these lines to understand the degree of compensation of these competing interac-

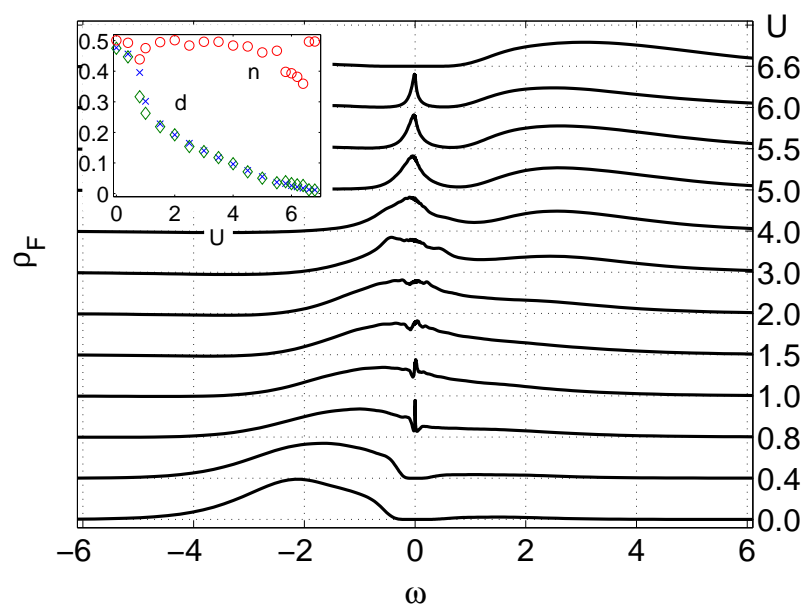

FIG. 12: Spectral density of the higher electronic Green's function $F(\omega)$. The weight for $\omega \leq 0$ indicates the degree of double occupancy; $g=0.45$ and various values of $U$. The inset shows the double occupancy calculated directly $(\times)$ and via the spectral theorem from $F(\omega)(\diamond)$. Circles indicate the total spectral weight of $F(\omega)$ which should evaluate to $n=1 / 2$ at half filling. The discrepancies are most pronounced near the phase transitions at $U \approx 1$ and $U \approx 6$.

tions for small phonon frequency $\omega_{0}=0.2$.

\section{A. Polaronic line $U_{\text {eff }}=0$}

Figure 13 shows the one-electron spectral functions for various values of $g$ and $U$ such that $U_{\text {eff }}=0$. For small values of $U$ (and $g$ ), we see the sharp feature in the electronic spectrum as discussed in Sec. II. However, upon increasing $U$ further, Hubbard bands develop and the central feature becomes the central quasiparti- 


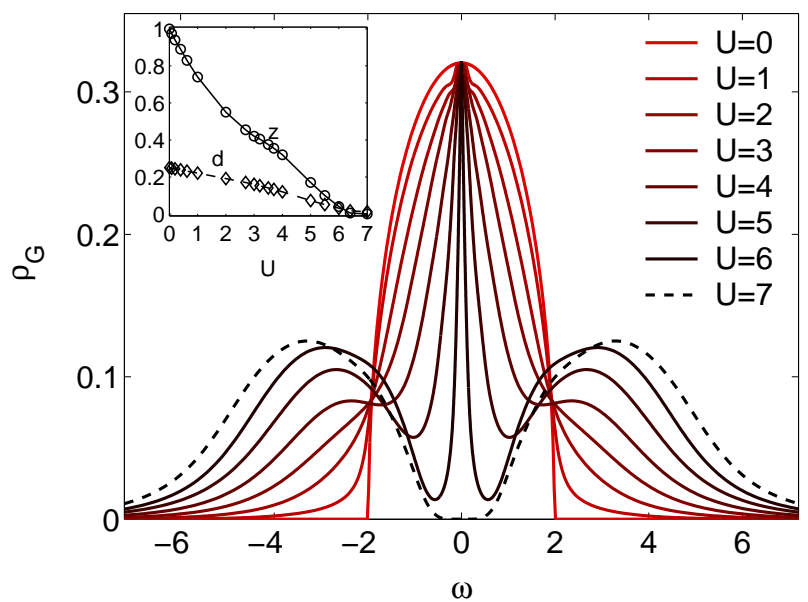

FIG. 13: One-particle spectral function on the line $U_{\text {eff }}=0$ at $\omega_{0}=0.2$ (dashed line in Fig. 1). The inset shows the variation of the quasiparticle weight $z$ and double occupancy $d=\left\langle n_{\uparrow} n_{\downarrow}\right\rangle$.

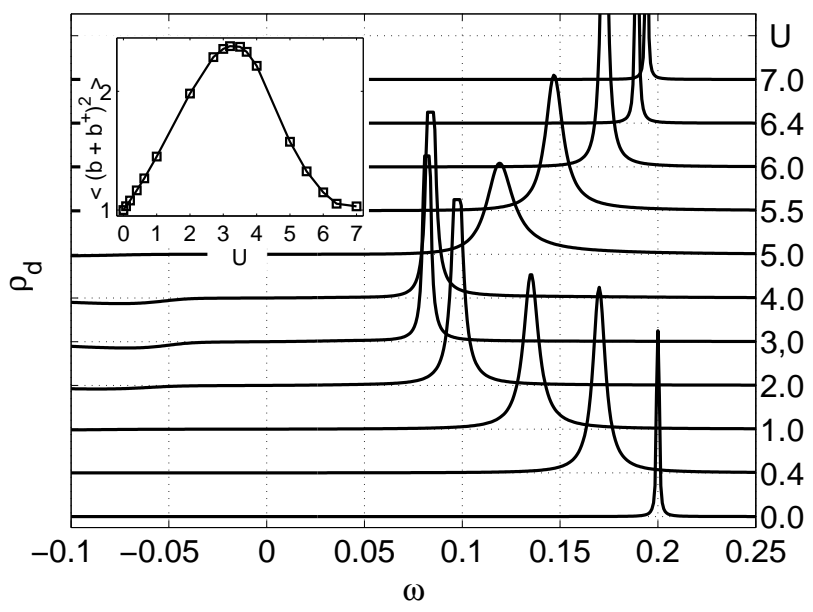

FIG. 14: Spectral density of the phonon propagator on various points on the polaronic line $U_{\text {eff }}=0$. The inset shows the expectation value of the lattice fluctuations as a function of $U$.

cle peak similar to the pure Hubbard model for large $U\left(<U_{c}\right)$. This indicates that phonons play their assumed role of compensating the Hubbard repulsion only to a very limited extent. The only remarkable difference to the pure Hubbard model is that on the line $U_{\text {eff }}=0$ the phase transition is shifted towards a higher value of $U_{c} \simeq 6.5$, as compared to $U_{\mathrm{c}}=5.88$ for $g=0$. The inset to Fig. 13 shows that the quasiparticle weight decreases steadily from $z=1$ to zero and the double occupancy from $\left\langle n_{\uparrow} n_{\downarrow}\right\rangle=1 / 4$ to a small but finite value.

The phonon spectra, as displayed in Fig. 14, are always made up of only one single peak that initially moves towards lower energies as more phonons are excited and lattice fluctuations increase (see inset). At $U \simeq 3$ this trend reverses and the phonon peak hardens back when

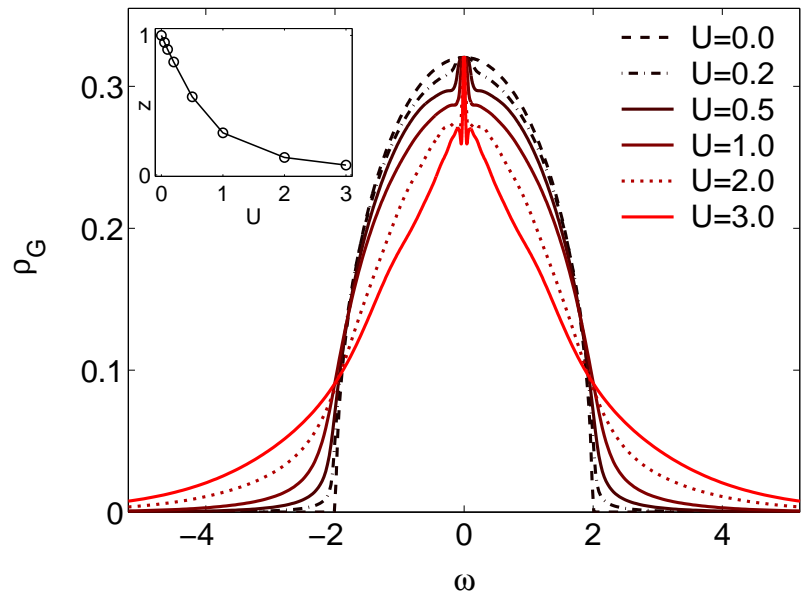

FIG. 15: One-particle spectral function on the line $\left\langle n_{\uparrow} n_{\downarrow}\right\rangle=$ 1/4. (dot-dashed line in Fig. 1). The inset shows the variation of the quasiparticle weight $z$ along this line.

approaching the phase transition to the gapped state. Simultaneously, the number of excited phonons decreases to zero and the lattice fluctuations reach the value of the quantum mechanical zero point fluctuations. The reason for this non-monotonic behaviour of the lattice fluctuations as function of $g$ is not obvious. The maximum in the lattice fluctuations as shown in the inset correlates with the value of $U$ where the transition to the bipolaronic state changes from second order (for $U \lesssim 3$ ) to first order (for $U \gtrsim 3$ ). This could be a possible explanation as one would expect to see stronger lattice fluctuations as one aproaches a second-order transition to the bipolaronic state.

\section{B. Locus of points with $\left\langle n_{\uparrow} n_{\downarrow}\right\rangle=1 / 4$}

The line in the phase diagram where $\left\langle n_{\uparrow} n_{\downarrow}\right\rangle=0.25$ starts at the uncorrelated system $(g=U=0)$ and ends at $U \approx 3$ where it merges with the phase boundary to the bipolaronic state. As a consequence, for larger values of $U$, the double occupancy has a jump at the transition from the metallic state $\left(\left\langle n_{\uparrow} n_{\downarrow}\right\rangle<0.25\right)$ to the bipolaronic state $\left(\left\langle n_{\uparrow} n_{\downarrow}\right\rangle \approx 0.5\right)$ (see Fig. 3 in Ref. 22 and inset of Fig. 6). Therefore this transition has to be first order in this range.

Figure 15 shows the one-electron spectral functions along this line. We observe the appearance of the narrow feature at the Fermi energy that is a typical for the electron-phonon coupling. The band develops broad shoulders, but they never separate into distinct subbands.

The phonon propagator, as displayed in Fig. 16 shows a remarkable softening of the original peak and the buildup of negative spectral weight. As a consequence, the lattice fluctuations increase almost linearly with $U$ and are quite pronounced for $U \rightarrow 3$. 


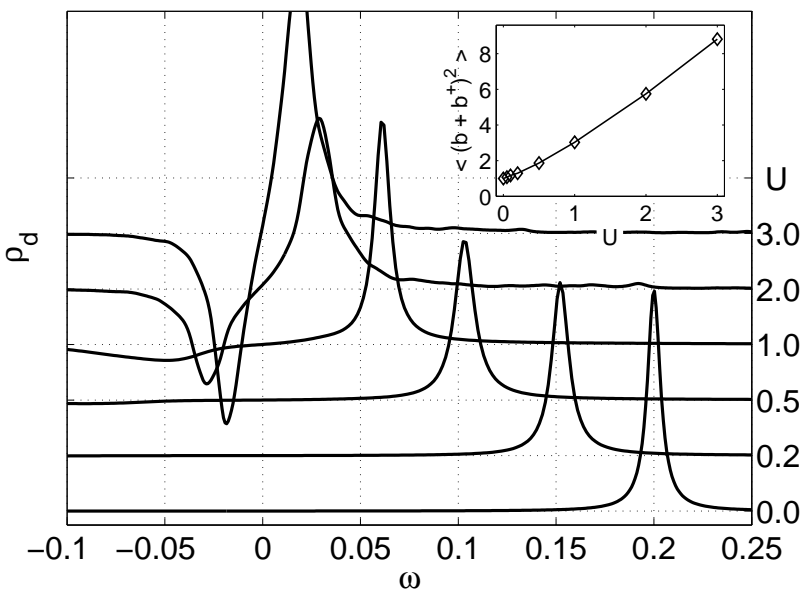

FIG. 16: Spectral density of the phonon propagator on various points on the line $\left\langle n_{\uparrow} n_{\downarrow}\right\rangle=1 / 4$. The inset shows the expectation value of the lattice fluctuations as a function of $U$ along this line.

\section{Dependence on $\omega_{0}$}

So far we have worked with a fixed value of $\omega_{0}=0.2$. However, the degree of compensation of the competing interactions is dependent on $\omega_{0}$. Here we return to the case $U_{\text {eff }}=0$ and study the variation of $\omega_{0}$ with fixed $U=5$ and $g=\sqrt{U \omega_{0} / 2}$.

In Fig. 17 we plot the one-electron spectral density for a range of values of $\omega_{0}$. For the largest value of $\omega_{0}=16$, the spectrum is virtually the same as that of the free system, because we have almost complete compensation. We now examine the behaviour as we progressively reduce $\omega_{0}$. First the high-energy phonon subbands gain weight and move towards the Fermi energy. Their position is roughly given by $\omega_{0}$. There is a commensurate reduction of the width of the central peak. For $\omega_{0}=2$ the

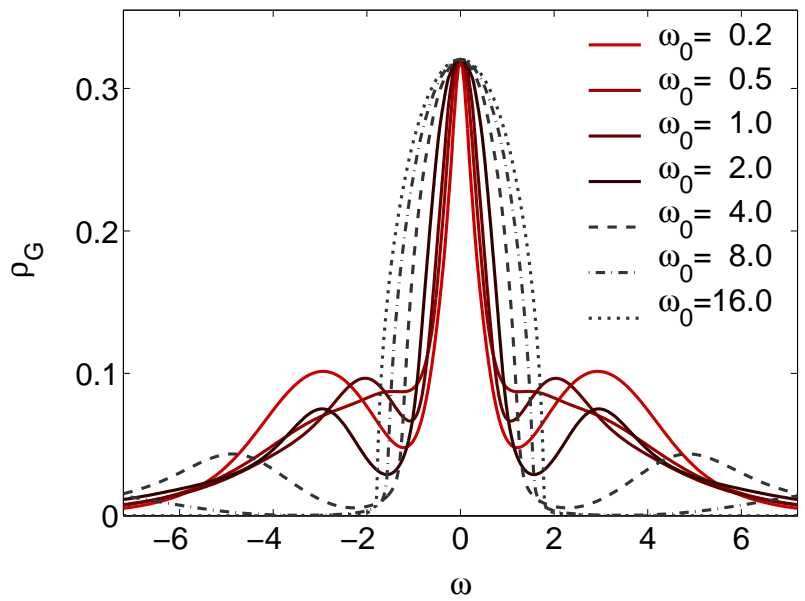

FIG. 17: One-particle spectral function for $U_{\text {eff }}=0, U=5$ and various values of $\omega_{0}$.

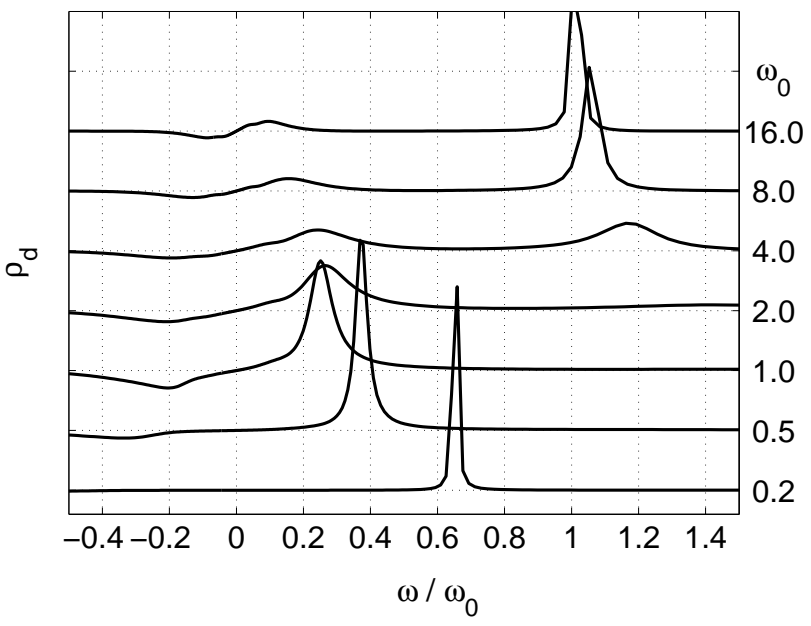

FIG. 18: Spectral density of the phonon propagator for $U_{\text {eff }}=$ 0 and $U=5$ for various values of $\omega_{0}$.

phonon subbands are visible as shoulders of the central peak. Upon further reduction of $\omega_{0}$, these shoulders move back towards higher energies again and, for $\omega_{0} \lesssim 0.5$, become the Hubbard bands located at $\omega \approx \pm U / 2= \pm 2.5$. For the smallest value of $\omega_{0}=0.2$, the spectrum is identical to the one discussed in Sec. IV A.

In Fig. 18 we plot the spectra of the phonon propagator as a function of the relative energy scale $\omega / \omega_{0}$. For large $\omega_{0}=16$, where the compensation is almost complete, we see the narrow phonon peak at $\omega_{0}$. In addition we see a small feature near $\omega=0$. As $\omega_{0}$ is decreased, the lowenergy feature gains weight and the mode at $\omega_{0}$ broadens and moves to slightly higher energies, as one would expect when $\omega_{0}$ approaches but lies above the upper band edge. At $\omega_{0}=4$, the two features have approximately equal weight. For smaller values of $\omega_{0}$ the upper peak has almost zero weight. The remaining peak is the soft mode discussed in the previous sections.

In Fig. 19 we illustrate how different static quantities reflect the trend of the Holstein-Hubbard model towards a free system in the limit of large $\omega_{0}$. We plot $z$ and $4\left\langle n_{\uparrow} n_{\downarrow}\right\rangle$ as a function of $\omega_{0}$. The double occupation value converges relatively rapidly to that of the free state. The quasiparticle weight, however, converges rather more slowly. In fact, only for $\omega_{0} \gg W=4$ is it approaching the uncorrelated limit $z=1$.

In the same Fig. 19 we also plot the lattice fluctuations $\left\langle x^{2}\right\rangle=\left\langle\left(b+b^{\dagger}\right)^{2}\right\rangle /\left(2 \omega_{0}\right)$. This curve goes monotonically to zero as $\omega_{0} \rightarrow \infty$ asymptotically behaving as $1 / \omega_{0}$ for large $\omega_{0}$. However, if we plot the rescaled values $\langle(b+$ $\left.\left.b^{\dagger}\right)^{2}\right\rangle$, we find a a maximum which coincides with the region of maximum softening of the phonon mode. For larger values of $\omega_{0}$ this quantity decreases to the quantum limit of $\left\langle\left(b+b^{\dagger}\right)^{2}\right\rangle=1$.

One would expect the phonons to decouple from the electrons in the limit of large $\omega_{0}$. It is interesting to observe, however, that $1+4\left\langle b^{\dagger} b\right\rangle$ (dot-dashed line in Fig. 19) fits well the high- $\omega_{0}$ behaviour of the lattice fluctuations 


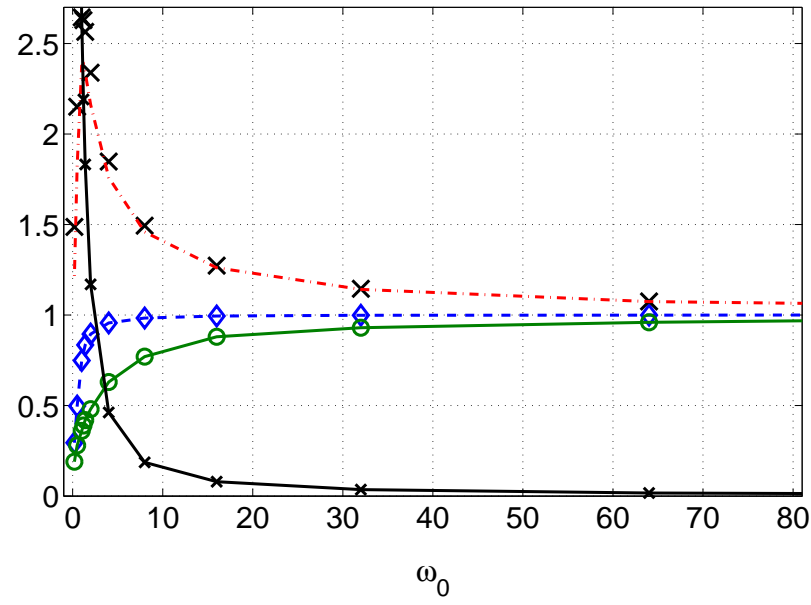

FIG. 19: Variation of the quasiparticle weight $z$ (o), the rescaled double occupancy $4\left\langle n_{\uparrow} n_{\downarrow}\right\rangle(\diamond)$, and lattice fluctuations $\left\langle x^{2}\right\rangle(\times)$ as a function of $\omega_{0}$ for $U_{\text {eff }}=0$. The dot-dashed line represents the number of excited phonons $1+4\left\langle b^{\dagger} b\right\rangle$.

which indicates that the ground state of the phonons in this limit is a coherent state rather than an eigenstate of the free phonon Hamiltonian $\omega_{0} b^{\dagger} b$.

\section{OVERVIEW}

For an overview we will find it useful to classify the metallic state in the phase diagram into three regions, separated by the two polaronic lines, In each of these we find qualitatively different behaviour.

We consider first of all the region below the line $U_{\text {eff }}=$ 0 . Here $U_{\text {eff }}>0$. One interesting question is how the coupling to the phonons affects the transition to the Mott insulating state. Our results indicate that this transition is always very similar to that found in the pure Hubbard model: the appearance of a preformed gap, a rather large coexistence region between insulating and metallic solutions and the quasiparticle weight continuously going to zero. There is only a small increase in the critical value of $U$ which is found empirically to depend upon $g$ to good approximation as

$$
U_{c} \approx U_{\mathrm{c}, \mathrm{Hubb}}+0.8 \times g^{2} .
$$

The small influence of the phonon coupling can be readily explained by suppression of charge fluctuations for large $U$. As the electron-phonon coupling in the HolsteinHubbard model is to the charge fluctuations, the suppression of these renders the coupling ineffective. In fact, the whole region $U_{\text {eff }}>0$ appears to be dominated by the Hubbard term. For $\omega \gg \omega_{0}$ the second term in the retarded interaction as defined in Eq. 2 is negligible and the effective interaction is essentially given by $U$. Also on the lowest energy scales $\omega \ll \omega_{0}, U_{\text {eff }}(\omega)>0$.

Next we look at the complementary region defined by $\left\langle n_{\uparrow} n_{\downarrow}\right\rangle>1 / 4$ which is dominated by the coupling to phonons. Here the similarity is with the pure Holstein model. The metal to bipolaronic transition takes place without a preformed gap and with the characteristic softening of the phonon mode. The transition in this region appears also to be continuous with no significant coexistence region. The main effect of the Hubbard $U$ is to push the phase transition and its precursors to somewhat larger values of $g$.

Finally, in the region bounded by the two curves $U_{\text {eff }}=0$ and $\left\langle n_{\uparrow} n_{\downarrow}\right\rangle=1 / 4$ there is more complex interplay of the two interactions, as can be seen in our results on the response functions. Beyond the point where the line $\left\langle n_{\uparrow} n_{\downarrow}\right\rangle=1 / 4$ merges into the phase boundary the transition to the bipolaronic state is first order and thus qualitatively different from that discussed in the last paragraph. Along the lower boundary line of this region, i.e., for $U_{\text {eff }}=0$, the effective interaction is still repulsive for $\omega \gg \omega_{0}$. The question arises whether on the lowest energy scale there is a complete cancellation of the two competing interactions. This question can be investigated by examining the lowest-lying one- and twoparticle excitations from the interacting ground state. The calculation of the effective interaction $\tilde{U}_{\text {eff }}$ between the renormalized quasiparticles has been considered in detail for impurity models in Ref. 29. A similar analysis is possible using the effective impurity model within the DMFT. From Ref. 29,

$$
\tilde{U}_{\text {eff }} \propto \lim _{N \rightarrow \infty}\left(E_{p p}(N)-2 E_{p}(N)\right) \Lambda^{(N-1) / 2},
$$

where $E_{p}(N)\left(E_{p p}(N)\right)$ is the energy of the lowest-lying single-particle (particle-particle) excitation in the $N$-th iteration of the NRG procedure and $\Lambda>1$ is the NRG discretization parameter. As for the impurity model, we find that, within the numerical accuracy of the NRG, $\tilde{U}_{\text {eff }}$ vanishes on the line $U_{\text {eff }}=0$ and is positive (negative) below (above) this line. Therefore, along this line we have a system of renormalized $(z<1)$ but non-interacting quasiparticles. On the high-energy scale $\omega>\omega_{0}$, the system is still dominated by $U$. This can be seen by the formation of the Hubbard bands as shown in Fig. 13 and the suppression of the double occupancy.

\section{CONCLUSIONS}

In this study we have investigated the particle-hole symmetric Holstein-Hubbard model. We have used the dynamical mean field theory in combination with the numerical renormalization group to calculate dynamical correlation functions for the full Hamiltonian with quantum phonons. This non-perturbative approach allows us to access all parameter regimes of the model at zero temperature. A non-perturbative technique is indeed necessary because perturbative methods such as Midgal-Eliashberg approach for the Holstein model are known to break down ${ }^{15}$ in the strong-coupling limit.

We find three regions with qualitatively different behaviour. In the region of the Mott metal insulator transi- 
tion we find the phonon effects are largely suppressed by the on-site repulsion $U$. This conclusion may be specific for the non-degenerate Holstein-Hubbard model where the coupling is purely to the local charge density. A system with orbital degeneracy and Jahn-Teller phonons shows $^{20}$ strong phonon effects even for strong $U$. In our case of the non-degenerate Holstein-Hubbard model, the electronic effects on phonons yield only a modest softening of the phonon mode in the metallic regime. This softening disappears in the Mott insulator.

Weak electron-electron interactions seem to have little effect other than to delay the onset of the transition to the bipolaronic phase. For larger values of $U$ the transition changes from continuous to discontinuous. For small values of $U$ we see a complete softening of the phonon peak when approaching the transition. The softening is much less pronounced for larger $U$.

The line $U_{\text {eff }}=0$ in the metallic phase does seem to have some significance in that for $U_{\text {eff }}>0$ the spin susceptibility has a dominant low-energy peak. For $U_{\text {eff }}<0$ the dominant low-energy peak is in the charge susceptibility. The line $U_{\text {eff }}=0$ also appears to be significant for the low-energy Fermi liquid behaviour as the on-site quasiparticle interaction changes sign on or close to this line. This is not obvious as one might have expected that on the low energy scale of the electron-phonon interaction, the electron-electron interaction is no longer given by the bare $U$ but some renormalized value $\bar{U}$. This would imply that one should use $\bar{U}$ in Eq. 2 on the energy scale $\omega \ll \omega_{0}$, so that the change of sign of the quasiparticle interaction $\tilde{U}_{\text {eff }}$, as defined in Eq. (8), would occur when $\bar{U}-2 g^{2} / \omega_{0}=0$. However, our results indicate that this is not the case and that $\tilde{U}_{\text {eff }}$ vanishes on or close to the line $U_{\text {eff }}=U-2 g^{2} / \omega_{0}=0$. A similar situation was found in the case the Anderson-Holstein impurity model $^{29}$.

The Holstein-Hubbard model is very rich and shows also diverse forms of behaviour that could not be addressed in this paper. Apart from the results presented here, this model is known to exhibit various types of symmetry-breaking phases ${ }^{17}$. We intend to extend our calculations to investigate the competition between charge order, antiferromagnetism, superconductivity and the effects of doping ${ }^{30}$. This should enable us to make direct contact with experimental results on such compounds as the fullerides, where this model with the assumption of the coupling of the electron density to the local phonon mode should be directly applicable. For applications to Jahn-Teller systems, such as in the manganites, the model would have to be extended to include degenerate orbitals.

\section{Acknowledgments}

We wish to thank the EPSRC (Grant GR/S18571/01) for financial support. This work was partially supported by SunnyNames llp. We acknowledge fruitful discussions with A. Gogolin, D. Edwards and Y. Ōno.

\section{APPENDIX A: PHONON PROPAGATOR AND $\chi_{c}\left(\omega_{0}\right)=0$}

Here we reconsider the relation between the phonon propagator and the charge susceptibility (Eq. (6)) for the Holstein-Hubbard model and comment on pitfalls in its exploitation.

Equations of motion: For the derivation of Eq. (6), we can use the standard equation of motion for the Fourier transform of the double-time Green's function,

$$
\omega\langle\langle A ; B\rangle\rangle_{\omega}=\left\langle[A, B]_{\eta}\right\rangle+\left\langle\left\langle[A, H]_{-} ; B\right\rangle\right\rangle_{\omega}
$$

For the boson Green's function $d(\omega)$ we take $A=b_{i}, B=$ $b_{i}^{\dagger}$, and $\eta=-1$. For $D(\omega)$ we take $A=B=b_{i}+b_{i}^{\dagger}$ and $\eta=-1$. The following procedure works for both $d(\omega)$ and $D(\omega)$, but we will use the former in what follows.

The equation of motion gives

$$
\left(\omega-\omega_{0}\right) d(\omega)=1+g\left\langle\left\langle\hat{O}_{i} ; b_{i}^{\dagger}\right\rangle\right\rangle_{\omega},
$$

where $\hat{O}_{i}=\sum_{\sigma} n_{i \sigma}-1$. Taking the equation of motion for the right-hand operator, we have

$$
\left(\omega-\omega_{0}\right)\left\langle\left\langle\hat{O}_{i} ; b_{i}^{\dagger}\right\rangle\right\rangle_{\omega}=g\left\langle\left\langle\hat{O}_{i} ; \hat{O}_{i}\right\rangle\right\rangle_{\omega} .
$$

Hence the result $\left(d_{0}(\omega)=\left(\omega-\omega_{0}\right)^{-1}\right.$ is the free phonon propagator),

$$
d(\omega)=d_{0}(\omega)+g^{2} d_{0}(\omega)\left\langle\left\langle\hat{O}_{i} ; \hat{O}_{i}\right\rangle\right\rangle_{\omega} d_{0}(\omega)
$$

which is Eq. (6) linking the phonon propagator $d(\omega)$ with the charge susceptibility $\chi_{c}(\omega)=\left\langle\left\langle\hat{O}_{i} ; \hat{O}_{i}\right\rangle\right\rangle_{\omega}$.

As a Green's function, the phonon propagator has a series of poles of first order, but none of second order. For this to be the case, Eq. (A4) tells us that the charge susceptibility has to vanish as

$$
\chi_{c}(\omega) \sim\left(\omega-\omega_{0}\right) \quad \text { for } \quad \omega \rightarrow \omega_{0}
$$

in order to ensure that the pole of $d(\omega)$ at $\omega=\omega_{0}$ is first order. This is also evident if we express $\chi_{c}(\omega)$ in terms of the irreducible particle-hole bubble $\Pi(\omega)$. It takes the form

$$
\chi_{c}(\omega)=\frac{\Pi(\omega)}{1-g^{2} \Pi(\omega) D_{0}(\omega)}
$$

with the non-interacting phonon propagator $D_{0}(\omega)=$ $2 \omega_{0} /\left(\omega^{2}-\omega_{0}^{2}\right)$. Since $D_{0}(\omega)$ diverges at $\omega=\omega_{0}$, we have $\chi_{c}\left(\omega_{0}\right)=0$.

Spectral Density: We will derive an expression for the spectral density of the phonon propagator, i.e., for

$$
\rho_{d}(\omega)=-\frac{1}{\pi} \lim _{\delta \rightarrow 0} d(\omega+i \delta)
$$


that implicitly includes the fact that $\chi_{c}\left(\omega_{0}\right)=0$.

Consider the system with a finite basis such that,

$$
\chi_{c}(\omega)=\sum_{j} \frac{\left|\alpha_{j}\right|^{2}}{\omega-\omega_{j}}
$$

for $\omega \geq 0$, where $\left\{\omega_{j}\right\}$ are the discrete set of excitations in the charge density response function. Then

$$
\frac{1}{\left(\omega-\omega_{0}+i \delta\right)^{2}} \chi_{c}(\omega+i \delta)=-\chi_{c}(\omega+i \delta) \frac{\partial}{\partial \omega} \frac{1}{\left(\omega-\omega_{0}+i \delta\right)}
$$

The contributions to the spectral density $\rho_{d}(\omega)$ arise solely from the poles at $\omega=\omega_{0}$ and $\omega=\omega_{j}$. These do not coincide because $\chi_{c}\left(\omega_{0}\right)=0$. The contribution to $\rho_{d}(\omega)$ from the poles at $\omega=\omega_{j}$ are straightforward to evaluate and give

$$
g^{2} \sum_{j} \frac{\left|\alpha_{j}\right|^{2}}{\left(\omega-\omega_{j}\right)^{2}} \delta\left(\omega-\omega_{j}\right)
$$

The contribution from the pole at $\omega=\omega_{0}$ is a little trickier to evaluate. The term in $g^{2}$ contributes

$$
-g^{2} \chi_{c}(\omega) \delta^{\prime}\left(\omega-\omega_{0}\right)
$$

where $\delta^{\prime}\left(\omega-\omega_{0}\right)$ denotes the derivative of the delta function. However, we have

$$
\chi_{c}(\omega) \delta^{\prime}\left(\omega-\omega_{0}\right)=-\chi_{c}^{\prime}\left(\omega_{0}\right) \delta\left(\omega-\omega_{0}\right)+\chi_{c}\left(\omega_{0}\right) \delta^{\prime}\left(\omega-\omega_{0}\right)
$$

where $\chi_{c}^{\prime}(\omega)$ is the derivative of $\chi_{c}(\omega)$ and is given by

$$
\chi_{c}^{\prime}(\omega)=-\sum_{j} \frac{\left|\alpha_{j}\right|^{2}}{\left(\omega-\omega_{j}\right)^{2}}
$$

Using $\chi_{c}\left(\omega_{0}\right)=0$ and collecting all the terms together we obtain

$$
\begin{aligned}
\rho_{d}(\omega)= & \left(1-g^{2} \sum_{j} \frac{\left|\alpha_{j}\right|^{2}}{\left(\omega_{0}-\omega_{j}\right)^{2}}\right) \delta\left(\omega-\omega_{0}\right) \\
& +g^{2} \sum_{j} \frac{\left|\alpha_{j}\right|^{2}}{\left(\omega_{0}-\omega_{j}\right)^{2}} \delta\left(\omega-\omega_{j}\right) .
\end{aligned}
$$

This expression can be used to calculate the spectral density of the phonon propagator $\rho_{d}(\omega)$ from the peaks $\left\{\alpha_{j}, \omega_{j}\right\}$ of the charge susceptibility $\chi_{c}(\omega)$. In fact, the numerical evaluation of Eq. (A14) yields the correct result for the phonon propagator and the lattice fluctuations, as shown in Fig. 5 for the case of the pure Holstein model. The condition $\chi_{c}\left(\omega_{0}\right)=0$ has, of course, been used explicitly in the derivation of Eq. (A14).

The problem of the direct evaluation of Eq. (A4) for the phonon propagator is the numerical error in $\chi_{c}(\omega)$. In fact, the truncation of the Hilbert space in the NRG procedure entails that the condition (A5) is not exactly met. Therefore $\chi_{c}(\omega)$ has no root exactly at $\omega=\omega_{0}$. This leads to a double pole of $d(\omega)$ at $\omega=\omega_{0}$ and to a distorted result for the phonon propagator at energies close to $\omega_{0}$, when derived from Eq. (A4). Lattice fluctuation, as calculated by integrating $D(\omega)$ from Eq. (A4) are strongly overestimated for the same reason.

\section{APPENDIX B: FORMULA FOR AVERAGE DISPLACEMENT $\langle\boldsymbol{x}\rangle$ :}

In this appendix we derive an expression linking the average local displacement $\left\langle x_{i}\right\rangle \sim\left\langle b_{i}^{\dagger}+b_{i}\right\rangle$ to the local electron density. For this derivation, we modify the Hamiltonian by coupling an extra term linear in $x_{i}$ at each site. The extra term in the Hamiltonian reads

$$
\sum_{i} \alpha_{i}\left(b_{i}^{\dagger}+b_{i}\right)
$$

with the coupling $\alpha \equiv\left\{\alpha_{i}\right\}$. Upon differentiating the expression for the partition function $Z$ with respect to $\alpha_{i}$, we obtain

$$
\left\langle b_{i}^{\dagger}+b_{i}\right\rangle=-\left.\frac{1}{Z \beta} \frac{\partial Z}{\partial \alpha_{i}}\right|_{\alpha=0} .
$$

We then apply a canonical transformation to the Hamiltonian, $\tilde{H}=\hat{U}^{-1} H \hat{U}$, with $\hat{U}$ given by

$$
\hat{U}=\prod_{i} e^{-\left(\alpha_{i}+g \hat{O}_{i}\right)\left(b_{i}^{\dagger}-b_{i}\right) / \omega_{0}} .
$$

This is a displaced oscillator transformation at each lattice site. The phonon and electron operators are transformed as

$$
\begin{gathered}
\tilde{b}_{i} \equiv \hat{U}^{-1} b_{i} \hat{U}=b_{i}-\frac{\alpha_{i}}{\omega_{0}}-\frac{g}{\omega_{0}} \hat{O}_{i}, \\
\tilde{c}_{i \sigma} \equiv \hat{U}^{-1} c_{i \sigma} \hat{U}=e^{-\frac{g}{\omega_{0}}\left(b_{i}^{\dagger}-b_{i}\right)} c_{i \sigma} .
\end{gathered}
$$

After some algebra we find that the terms depending on $\alpha$ in the transformed Hamiltonian $\tilde{H} \equiv \hat{U}^{-1} H \hat{U}$ read

$$
-\sum_{i}\left(\frac{\alpha_{i}^{2}}{\omega_{0}}+2 \alpha_{i} \frac{g}{\omega_{0}} \hat{O}_{i}\right) .
$$

The partition function $Z$ is, of course, not changed by the canonical transformation of the Hamiltonian. Upon expressing $Z$ in terms of $\tilde{H}$, differentiating with respect to $\alpha_{i}$ and then putting $\alpha=0$ we find the result

$$
\left\langle b_{i}^{\dagger}+b_{i}\right\rangle=-\frac{2 g}{\omega_{0}}\left\langle\sum_{\sigma} n_{i \sigma}-1\right\rangle,
$$

which relates the average local displacement to the local average electron density. 
* Electronic address: w.koller@imperial.ac.uk

$\dagger$ Electronic address: d.meyer@imperial.ac.uk

\# Electronic address: a.hewson@imperial.ac.uk

1 P. Fulde, J. Keller, and G. Zwicknagl, in Solid State Physics 41, edited by H. Ehrenreich and D. Turnbull (Academic Press, San Diego, 1988), pp. 1-150.

2 A. J. Millis, P. B. Littlewood, and B. I. Shraiman, Phys. Rev. Lett. 74, 5144 (1995).

3 D. M. Edwards, Adv. Phys. 51, 1259 (2002).

4 M. Imada, A. Fujimori, and Y. Tokura, Rev. Mod. Phys. 70, 1039 (1998).

${ }^{5}$ O. Gunnarsson, Rev. Mod. Phys. 69, 575 (1997).

6 A. Georges, G. Kotliar, W. Krauth, and M. J. Rozenberg, Rev. Mod. Phys. 68, 13 (1996).

7 J. Hubbard, Proc. R. Soc. London, Ser. A 276, 238 (1963).

${ }^{8}$ M. J. Rozenberg, X. Y. Zhang, and G. Kotliar, Phys. Rev. Lett. 69, 1236 (1992).

9 M. Jarrell, Phys. Rev. Lett. 69, 168 (1992).

10 T. Holstein, Ann. Phys. 8, 325 (1959).

11 J. K. Freericks, M. Jarrell, and D. J. Scalapino, Phys. Rev. B 48, 6302 (1993).

12 M. Capone and S. Ciuchi, Phys. Rev. Lett. 91, 186405 (2003).

13 J. K. Freericks, Phys. Rev. B 50, 403 (1994).

14 J. K. Freericks and M. Jarrell, Phys. Rev. B 50, 6939 (1994).

15 J. P. Hague and N. d'Ambrumenil, cond-mat/0106355.

16 D. Meyer, A. C. Hewson, and R. Bulla, Phys. Rev. Lett.
89, 196401 (2002).

17 J. K. Freericks and M. Jarrell, Phys. Rev. B 75, 2570 (1995).

18 J. E. Han and O. Gunnarsson, Phys. Rev. B 61, 8628 (2000).

19 A. Deppeler and A. J. Millis, Phys. Rev. B 65, 224301 (2002).

20 J. E. Han and O. Gunnarsson, Physica B 292, 196 (2000).

21 M. Capone, G. Sangiovanni, C. Castellani, C. D. Castro, and M. Grilli, Phys. Rev. Lett. 92, 106401 (2004).

${ }^{22}$ W. Koller, D. Meyer, Y. Ono, and A. C. Hewson, Europhys. Lett., in print, cond-mat/0312367.

23 G. S. Jeon, T.-H. Park, J. H. Han, H. C. Lee, and H.-Y. Choi, cond-mat/0312390.

24 A. C. Hewson and D. Meyer, J. Phys.: Condens. Matter 14, $427(2002)$.

${ }^{25}$ H. R. Krishna-murthy, J. W. Wilkins, and K. G. Wilson, Phys. Rev. B 21, 1003 (1980).

26 R. Bulla, Phys. Rev. Lett. 83, 136 (1999).

27 R. Bulla, A. C. Hewson, and T. Pruschke, J. Phys.: Condens. Matter 10, 8365 (1998).

28 G. S. Jeon, T.-H. Park, and H.-Y. Choi, Phys. Rev. B 68, 045106 (2003).

29 A. C. Hewson, A. Oguri, and D. Meyer, condmat/0312484.

$30 \mathrm{Y}$. Ono, in preparation. 